HUTP-01/A012

hep-th/0103080

CTP-MIT-3094

CTP TAMU-09/01

\title{
Supergravity duals of gauge theories from $F(4)$ gauged supergravity in six dimensions
}

\author{
Carlos Núñez ${ }^{a}$, I.Y. Park ${ }^{b} \dagger$, Martin Schvellinger ${ }^{c}$, Tuan A. Tran ${ }^{b_{\ddagger}}$ \\ ${ }^{a}$ Lyman Laboratory of Physics, \\ Harvard University, \\ Cambridge, Massachusetts 02138, USA \\ E-mail: nunez@lorentz.harvard.edu \\ ${ }^{b}$ Center for Theoretical Physics, \\ Texas A\&M University \\ College Station, Texas 77843, USA \\ †E-mail: ipark@rainbow.physics.tamu.edu \\ $\ddagger$ E-mail: tuan@rainbow.physics.tamu.edu \\ ${ }^{c}$ Center for Theoretical Physics, \\ Laboratory for Nuclear Science and Department of Physics, \\ Massachusetts Institute of Technology, \\ Cambridge, Massachusetts 02139, USA \\ E-mail: martin@ctpbeaker.mit.edu
}

\begin{abstract}
We construct supergravity solutions dual to twisted field theories that are the worldvolume theories of D4-branes wrapped on 2, 3-cycles, and NS-fivebranes on 2-cycles. We first obtain the solutions for the Romans' six-dimensional gauged supergravity theories and then up-lift them to ten dimensions. In particular, we find solutions for field configurations with either non-Abelian fields or $B$-fields being excited. One of these solutions, in the massless case, is up-lifted to the massless type IIA string theory. This is the first example of such a kind. The cases studied provide new examples of the AdS/CFT duality involving twisted field theories.
\end{abstract}




\section{Introduction}

The large $N$-limit of superconformal field theories have dual descriptions in terms of weakly coupled supergravities $[1,2,3]$. Generalizations to non-conformal cases were also studied [4]. There is an extensive list of references regarding the flows of $\mathcal{N}=4$ super Yang-Mills theory under the perturbations of either masses or vev's for bilinear operators $[5,6]$. With the twisted gauge field theories [7] available, it is possible to find new examples of AdS/CFT where only a part of the maximal supersymmetries is preserved $[8,9,10,11,12,13]$. This allows us to investigate supergravity duals of partially twisted gauge field theories. Therefore, wrapped fivebranes and D3-branes on holomorphic curves were studied in [8,9]. Similarly, fivebranes [10] and D3-branes [12] wrapped on associative three-cycles were considered. These studies have been extended to M-fivebranes wrapping Kähler 4-cycles, special Lagrangian 3, 4 and 5-cycles, coassociative 4-cycles and Cayley 4-cycles [11]. In this paper, we concentrate on the D4D8 system, which was studied in depth from the gauge theory side in ref. [14] and from the gravity/CFT viewpoint in refs. [15, 16]. We will wrap D4-branes of a D4-D8 system on two and three-cycles. We will also study solutions corresponding to smeared NSfivebranes wrapped on two-cycles. We first find solutions of six-dimensional Romans' gauged supergravity [17] and up-lift them to massive type IIA string theory. Other constructions in massive type IIA supergravity can be found in ref. [18], where some intersecting-brane configurations of D0-branes, D8-branes and a string were studied. Then, we analyze their dual twisted field theories and study the flows from $A d S_{6}$-type regions to the $A d S_{6-p} \times \Sigma_{p}$ solutions, where $\Sigma_{p}$ is the $p$-dimensional curved manifold. In the present case we consider $p=2$ and 3 .

The Romans' theories we are interested in are the $S U(2)$ gauged $\mathcal{N}=4$ supergravities in six dimensions. There are four different theories depending upon the values of the $S U(2)$ coupling constant $g$ and the mass parameter $m$ of the two-form field $B$. One of these theories has two anti-de Sitter vacua, one of them breaking all the supersymmetry and the other one preserving all of it. The latter theory, with a ground state with unbroken $F(4)$ supersymmetry, is the six-dimensional supergravity predicted in $[19,20]$. In this paper, we will consider this case.

We start out to find solutions of six-dimensional gauged supergravity with vanishing $B$-fields, using a hyperbolic metric ansatz of the type $A d S_{4} \times H_{2}$ (and also a spherical one, for $A d S_{4} \times S^{2}$ ). We find a fixed-point solution in the massive case, whereas for massless field configurations we find a general solution. The latter has "bad" type singularities: in order to resolve the singularities we look for solutions with additional degrees of freedom in a similar way to ref. [9]. This leads us to look for non-Abelian solutions. Another possible way to resolve singularities is to find a black hole solution where the singularity is covered by a horizon [21]. The aim of this paper is to find the 
above mentioned gravity solutions, perform the up-lifting of them to ten dimensions and, to study their dual (twisted) gauge field theories. Massive type IIA supergravity is an interesting theory which seems to be the most suitable arena for realizing de Sitter compactifications or Randall-Sundrum models [22] on manifolds with boundary. Therefore, the study of the systems like the ones we deal with here could give some ideas on general structures.

Since in order to study the flow between a higher dimensional theory and the resulting lower dimensional one it is necessary to compactify some of the directions on a curved manifold, spinors have to be defined on non-flat spaces. Therefore, this requires to deal with a twisted gauge field theory living in such a manifold. Thus, in order to consider the dual field theories corresponding to certain supergravity solutions in curved spaces, one has to study a twisted version of the corresponding supersymmetric theory. This new picture emerges from the impossibility in defining covariantly constant spinors in the aforementioned manifolds. Then, it is necessary to introduce a new term coming from the gauging of the normal bundle to the brane world-volume with the spin connection.

We consider spacetimes with geometries given by $A d S_{4} \times \Sigma_{2}$ and $A d S_{3} \times \Sigma_{3}$, such that their UV limit is an $A d S_{6}$-type spacetime. These lead to twisted field theories since we assume $\Sigma_{p}$ 's are curved manifolds. The decoupling limit is taken in such a way that it preserves the volume of the compact manifold. Then, the twisted field theory on the brane world-volume will be $R^{1,4-p} \times \Sigma_{p}$, for D4-branes wrapping $\Sigma_{2}$ and $\Sigma_{3}$. The field theory in this decoupling limit will not be sensitive to the global geometry of $\Sigma_{p}$, remaining its effect local and determining the twisted field theory [8]. For energies much lower than the radius of the compact manifold the theory will be a 3 (or 2) dimensional field theory, respectively.

This paper is organized as follows. In section 2 we review the basic formalism and set-up of the six-dimensional Romans' theories. Section 3 is devoted to the study of the gravity duals of superconformal field theories in 3 and 5 dimensions. We focus on a non-Abelian solution, which will be up-lifted to massive type IIA string theory. It can be interpreted as a D4-D8 system where the D4-brane is wrapped on 2- and 3-cycles. Section 4 begins with the up-lifting for the massless case to type IIA theory (even when expected this is another new result of this work). Then, we study the corresponding twisted gauge field theories. We discuss the relation between our solutions and the previous ones [10], regarding the structure of their actions in the string frame. Finally, we present our conclusions. 


\section{The Romans' theories in 6 dimensions}

In this section we review the six-dimensional gauged $\mathcal{N}=4$ supergravity constructed by Romans [17], whose conventions we follow. The theory consists of a graviton $e_{\mu}^{\alpha}$, three $S U(2)$ gauge potentials $A_{\mu}^{I}$, an Abelian potential $\mathcal{A}_{\mu}$, a two-index tensor gauge field $B_{\mu \nu}$, a scalar $\phi$, four gravitinos $\psi_{\mu i}$ and four gauginos $\chi_{\mu}$. The bosonic Lagrangian is

$$
\begin{aligned}
e^{-1} \mathcal{L}= & -\frac{1}{4} R+\frac{1}{2}\left(\partial^{\mu} \phi\right)\left(\partial_{\mu} \phi\right)-\frac{1}{4} \mathrm{e}^{-\sqrt{2} \phi}\left(\mathcal{H}^{\mu \nu} \mathcal{H}_{\mu \nu}+F^{I \mu \nu} F_{\mu \nu}^{I}\right) \\
& +\frac{1}{12} \mathrm{e}^{2 \sqrt{2} \phi} G_{\mu \nu \rho} G^{\mu \nu \rho}+\frac{1}{8}\left(g^{2} \mathrm{e}^{\sqrt{2} \phi}+4 g m \mathrm{e}^{-\sqrt{2} \phi}-m^{2} \mathrm{e}^{-3 \sqrt{2} \phi}\right) \\
& -\frac{1}{8} e \varepsilon^{\mu \nu \rho \sigma \tau \kappa} B_{\mu \nu}\left(\mathcal{F}_{\rho \sigma} \mathcal{F}_{\tau \kappa}+m B_{\rho \sigma} \mathcal{F}_{\tau \kappa}+\frac{1}{3} m^{2} B_{\rho \sigma} B_{\tau \kappa}+F_{\rho \sigma}^{I} F_{\tau \kappa}^{I}\right),
\end{aligned}
$$

where $e$ is the determinant of the vielbein, $g$ is the $S U(2)$ coupling constant, $m$ is the mass parameter associated with the two-index tensor field $B_{\mu \nu}$ and $\varepsilon_{\mu \nu \rho \sigma \tau \kappa}$ is a LeviCivita tensor density. The Abelian field strength $\mathcal{F}_{\mu \nu}$, the non-Abelian one $F_{\mu \nu}^{I}$, the three-form $G_{\mu \nu \rho}$ and the field $\mathcal{H}_{\mu \nu}$ are given by

$$
\begin{aligned}
\mathcal{F}_{\mu \nu} & \equiv \partial_{\mu} \mathcal{A}_{\nu}-\partial_{\nu} \mathcal{A}_{\mu}, \\
F_{\mu \nu}^{I} & \equiv \partial_{\mu} A_{\nu}^{I}-\partial_{\nu} A_{\mu}^{I}+g \epsilon^{I J K} A_{\mu}^{J} A_{\nu}^{K}, \\
G_{\mu \nu \rho} & =3 \partial_{[\mu} B_{\nu \rho]}, \\
\mathcal{H}_{\mu \nu} & \equiv \mathcal{F}_{\mu \nu}+m B_{\mu \nu},
\end{aligned}
$$

respectively. The supersymmetry transformations for the gauginos and gravitinos are

$$
\begin{aligned}
\delta \chi_{i}= & \left(\frac{1}{\sqrt{2}} \gamma^{\mu} \partial_{\mu} \phi+A \gamma_{7}-\frac{1}{12} \mathrm{e}^{\sqrt{2} \phi} \gamma_{7} \gamma^{\mu \nu \rho} G_{\mu \nu \rho}\right) \epsilon_{i}+\frac{1}{2 \sqrt{2}} \gamma^{\mu \nu}\left(\hat{H}_{\mu \nu}\right)_{i}{ }^{j} \epsilon_{j} \\
\delta \psi_{\mu i}= & \left(\nabla_{\mu}+T \gamma_{\mu} \gamma_{7}-\frac{1}{24} \mathrm{e}^{\sqrt{2} \phi} \gamma_{7} \gamma^{\nu \rho \sigma} G_{\nu \rho \sigma} \gamma_{\mu}\right) \epsilon_{i} \\
& +\left(g A_{\mu}^{I}\left(T^{I}\right)_{i}{ }^{j}-\frac{1}{4 \sqrt{2}}\left(\gamma_{\mu}{ }^{\nu \rho}-6 \delta_{\mu}{ }^{\nu} \gamma^{\rho}\right)\left(\hat{H}_{\nu \rho}\right)_{i}{ }^{j}\right) \epsilon_{j}
\end{aligned}
$$

where $A, T$, and $\hat{H}$ are defined as follows

$$
\begin{aligned}
A & \equiv \frac{1}{4 \sqrt{2}}\left(g \mathrm{e}^{\frac{\phi}{\sqrt{2}}}-3 m \mathrm{e}^{\frac{-3 \phi}{\sqrt{2}}}\right), \quad T \equiv-\frac{1}{8 \sqrt{2}}\left(g \mathrm{e}^{\frac{\phi}{\sqrt{2}}}+m \mathrm{e}^{\frac{-3 \phi}{\sqrt{2}}}\right), \\
\left(\hat{H}_{\mu \nu}\right)_{i}{ }^{j} & \equiv \mathrm{e}^{-\frac{\phi}{\sqrt{2}}}\left(\frac{1}{2} \mathcal{H}_{\mu \nu} \delta_{i}{ }^{j}+\gamma_{7} F_{\mu \nu}^{I}\left(T^{I}\right)_{i}{ }^{j}\right) .
\end{aligned}
$$

The gauge-covariant derivative $\mathcal{D}_{\mu}$ acting on the Killing spinor is

$$
\mathcal{D}_{\mu} \epsilon_{i}=\nabla_{\mu} \epsilon_{i}+g A_{\mu}^{I}\left(T^{I}\right)_{i}^{j} \epsilon_{j}
$$


with

$$
\nabla_{\mu} \epsilon_{i} \equiv\left(\partial_{\mu}+\frac{1}{4} \omega_{\mu}^{\alpha \beta} \gamma_{\alpha \beta}\right) \epsilon_{i}
$$

where $\omega_{\mu}^{\alpha \beta}$ is the spin connection. Indices $\alpha, \beta$ are tangent space (or flat) indices, while $\mu, \nu$ are spacetime (or curved) indices. The $\gamma_{\alpha \beta \ldots}$ are the six-dimensional Dirac matrices,

$$
\gamma_{\alpha_{1} \cdots \alpha_{n}}=\frac{1}{n !} \gamma_{\left[\alpha_{1}\right.} \cdots \gamma_{\left.\alpha_{n}\right]}, \quad n=1, \cdots, 6 .
$$

The equations of motion of the Lagrangian (1) are

$$
\begin{aligned}
R_{\mu \nu}= & 2 \partial_{\mu} \phi \partial_{\nu} \phi+\frac{1}{8} g_{\mu \nu}\left(g^{2} \mathrm{e}^{\sqrt{2} \phi}+4 g m \mathrm{e}^{-\sqrt{2} \phi}-m^{2} \mathrm{e}^{-3 \sqrt{2} \phi}\right) \\
& +\mathrm{e}^{2 \sqrt{2} \phi}\left(G_{\mu}{ }^{\rho \sigma} G_{\nu \rho \sigma}-\frac{1}{6} g_{\mu \nu} G^{\rho \sigma \tau} G_{\rho \sigma \tau}\right) \\
& -2 \mathrm{e}^{-\sqrt{2} \phi}\left(\mathcal{H}_{\mu}{ }^{\rho} \mathcal{H}_{\nu \rho}-\frac{1}{8} g_{\mu \nu} \mathcal{H}_{\rho \sigma} \mathcal{H}^{\rho \sigma}\right) \\
& -2 \mathrm{e}^{-\sqrt{2} \phi}\left(F_{\mu}^{I \rho} F_{\nu \rho}^{I}-\frac{1}{8} g_{\mu \nu} F_{\rho \sigma}^{I} F^{I \rho \sigma}\right), \\
\square \phi= & \frac{1}{4 \sqrt{2}}\left(g^{2} \mathrm{e}^{\sqrt{2} \phi}-4 m g \mathrm{e}^{-\sqrt{2} \phi}+3 m^{2} \mathrm{e}^{-3 \sqrt{2} \phi}\right)+\frac{1}{3 \sqrt{2}} \mathrm{e}^{2 \sqrt{2} \phi} \\
& \times G^{\mu \nu \rho} G_{\mu \nu \rho}+\frac{1}{2 \sqrt{2}} \mathrm{e}^{-\sqrt{2} \phi}\left(\mathcal{H}^{\mu \nu} \mathcal{H}_{\mu \nu}+F^{I \mu \nu} F_{\mu \nu}^{I}\right), \\
\mathcal{D}_{\nu}\left(\mathrm{e}^{-\sqrt{2} \phi} \mathcal{H}^{\nu \mu}\right)= & \frac{1}{6} e \varepsilon^{\mu \nu \rho \sigma \tau \kappa} \mathcal{H}_{\nu \rho} G_{\sigma \tau \kappa}, \\
\mathcal{D}_{\nu}\left(\mathrm{e}^{-\sqrt{2} \phi} F^{I \nu \mu}\right)= & \frac{1}{6} e \varepsilon^{\mu \nu \rho \sigma \tau \kappa} F_{\nu \rho}^{I} G_{\sigma \tau \kappa}, \\
\mathcal{D}_{\rho}\left(\mathrm{e}^{2 \sqrt{2} \phi} G^{\rho \mu \nu}\right)= & -m \mathrm{e}^{-\sqrt{2} \phi} \mathcal{H}^{\mu \nu}-\frac{1}{4} e \varepsilon^{\mu \nu \rho \sigma \tau \kappa}\left(\mathcal{H}_{\rho \sigma} \mathcal{H}_{\tau \kappa}+F_{\rho \sigma}^{I} F_{\tau \kappa}^{I}\right) .
\end{aligned}
$$

Depending upon the values of the gauge coupling and mass parameter, there are five distinct theories: $\mathcal{N}=4^{+} \quad($ for $g>0, m>0), \quad \mathcal{N}=4^{-} \quad($ for $g<0, m>0), \quad \mathcal{N}=4^{g}$ (for $g>0, m=0), \quad \mathcal{N}=4^{m} \quad($ for $g=0, m>0)$, and $\mathcal{N}=4^{0} \quad($ for $g=0, m=0)$. The $\mathcal{N}=4^{g}$ theory coincides with a theory [23] obtained by dimensional reduction of gauged $\mathcal{N}=2$ supergravity in seven dimensions, followed by truncation. It was pointed out in [17] that there is a dual version of the $\mathcal{N}=4^{g}$ theory, which has a similar field content but cannot be obtained from the $\mathcal{N}=4^{g}$ theory by a field redefinition. The $B_{\mu \nu}$ field is replaced by a new tensor field $A_{\mu \nu}$, whose field strength is $\tilde{F}_{\mu \nu}^{I}$. In absence of the Abelian field strength, the bosonic Lagrangian of the dual $\mathcal{N}=\tilde{4}^{g}$ theory is

$$
\tilde{e}^{-1} \tilde{\mathcal{L}}=-\frac{1}{4} \tilde{R}+\frac{1}{2} \partial_{\mu} \tilde{\phi} \partial^{\mu} \tilde{\phi}-\frac{1}{4} \mathrm{e}^{-\sqrt{2} \tilde{\phi}} \tilde{F}_{\mu \nu}^{I} \tilde{F}^{I \mu \nu}+\frac{1}{12} \mathrm{e}^{-2 \sqrt{2} \tilde{\phi}} \tilde{F}_{\mu \nu \rho} \tilde{F}^{\mu \nu \rho}+\frac{1}{8} \tilde{g}^{2} \mathrm{e}^{\sqrt{2} \tilde{\phi}}
$$

where

$$
\tilde{F}_{\mu \nu \rho} \equiv 3\left(\partial_{[\mu} A_{\nu \rho]}-F_{[\mu \nu}^{I} A_{\rho]}^{I}-\frac{1}{3} g \epsilon^{I J K} A_{\mu}^{I} A_{\nu}^{J} A_{\rho}^{K}\right)
$$


The Lagrangian (14) can be obtained from the Lagrangian (1) by formally writing $\tilde{F}_{\mu \nu \rho}$ as follows

$$
\tilde{F}_{\mu \nu \rho}=\frac{1}{6} \mathrm{e}^{2 \sqrt{2} \tilde{\phi}} e \varepsilon_{\mu \nu \rho \sigma \tau \kappa} G^{\sigma \tau \kappa} .
$$

Since the Lagrangian (14) differs from $\mathcal{N}=4^{g}$ theory only by a sign of dilaton coupling to three-form field, in the absence of the three-form field $\mathcal{N}=4^{g}$ and $\mathcal{N}=\tilde{4}^{g}$ are identical. Furthermore, the Eq. (16) tells us that any solution of $\mathcal{N}=4^{g}$ theory with the two-form fields not being excited, which is also a solution of $\mathcal{N}=\tilde{4}^{g}$ theory, can be up-lifted into higher dimensional theory. We will consider the ten-dimensional interpretation of $\mathcal{N}=4^{g}$ theory in section 4 .

\section{Duals of 5 and 3-dimensional SCFTs}

In this section we study the gravity duals of superconformal field theories in five dimensions with 8 supercharges [14], which in the IR flow to superconformal field theories in three dimensions with 4 supercharges, which is $\mathcal{N}=2$ in three dimensions $[24,25]$.

The spinors of $S O(1,4)$ are pseudoreal with four components. These theories in 5 dimensions are related by compactifications to $\mathcal{N}=2$ four-dimensional theories. They have an $S U(2)_{R}$ symmetry of automorphism algebra which can be associated with the gauged symmetry in the gravitational set-up of [17]. The vector multiplet in five dimensions has one real scalar component, a vector gauge field and a spinor, while the hypermultiplet has four real scalars and a fermion. This theory has a Coulomb branch when the real scalar has a vev, and a Higgs branch when the scalar in the hypermultiplet is excited. Our gravitational system can be understood as follows. Suppose that we start from type I theory on $R^{9} \times S^{1}$, and we consider N D5-branes, the gauge theory on the branes is $\mathcal{N}=1 S p(N)$ gauge field theory with one hypermultiplet in the antisymmetric representation and 16 hypermultiplets in the fundamental. After Tdualizing on $S^{1}$ we arrive at a type I' configuration on $S^{1} / Z_{2}$ with two O8 planes, N D4-branes and 16 D8-branes. The hypermultiplet in the antisymmetric representation is massless while the mass of the fundamental hypermultiplets is given by the relative position of the D8-branes with respect to the D4-brane. The theory on the D4-brane is $S p(N)$ with one vector multiplet (whose scalar component describes the Coulomb branch $R^{+}$) and hypermultiplets whose first components describe the Higgs branch. The global symmetries of the theory are $S U(2)_{R} \times S U(2) \times S O\left(2 N_{f}\right) \times U(1)$, being the first $S U(2)$ the R-symmetry (the supercharges and the scalars in the hypermultiplets are doublets under this group). The second one is associated with the hypermultiplet in the antisymmetric representation and the rest is associated with the hypermultiplets in the fundamental and instantons. When the D4-brane is in the origin of the Coulomb branch we have a fixed point and the global symmetry is enhanced to $S U(2) \times E_{N_{f}+1}$. 
The gravitational system is given by $N_{f}$ D8-branes situated on a $O_{8}$ plane, $16-N_{f}$ D8-branes in another fixed plane and, a D4-brane which can move between them. The position of the D4-brane is parameterized by the scalar in the vector multiplet. If $\langle\phi\rangle$ is not zero we have a theory with a $U(1)$ symmetry with $N_{f}$ "electrons", on the fixed planes, the theory recovers its $S U(2)$ R-symmetry and it will have $N_{f}$ quarks.

The gravity theory is a fibration of $A d S_{6}$ over $S^{4}$, with isometries $S O(2,5) \times S U(2) \times$ $S U(2)$. The six-dimensional $S U(2)$ gauged supergravity has an $A d S_{6}$ vacuum solution. It can be up-lifted to massive IIA theory [26] leading to the configuration described above [12]. In our case we will consider solutions of Romans' theory which, when uplifted, will give ten dimensional configurations with $N_{f}=0$. These theories were very well studied in [30] in the context of algebraic geometry. There the authors studied the moduli spaces of type I' string and Heterotic string. It will be interesting to understand the case of $N_{f} \neq 0$ and see whether there is a gravity solution dual to the theory with enhanced symmetry $E_{1}^{\prime}$. It will be also useful to clarify the role of D0-branes in our gravity solutions. In principle they should correspond to excitations of the six-dimensional Abelian gauge field. We will not consider these fields here. We leave this for a future work.

In order to analyze the flow of the theory from five dimensions to a superconformal theory in three dimensions with four supercharges, we would like to consider a configuration where the geometry "loses" two dimensions at low energies. Therefore, the idea is the following: we start with a six-dimensional gauged supergravity theory having an $A d S_{6}$ vacuum and 8 preserved supercharges. This vacuum solution is dual to the fivedimensional SCFT mentioned above and its ten-dimensional interpretation is given by a D4-D8 system. When one moves to the IR of the gauge theory (flowing in the radial coordinate on the gravity dual) two of the dimensions of the theory become very small and no low energy massless modes are excited on this two-space. Therefore, effectively the gauge theory is three-dimensional. Besides, since the D4-brane is wrapped on a curved surface, we need to twist the theory. The effect of this twisting is the breaking of some supersymmetries, as it can be seen from the projections of the Killing vectors.

Under the group $S O(1,4) \times S O(3)_{R}$ the fields on the D4-brane transform as $(\mathbf{1}, \mathbf{1})$, $(\mathbf{3}, \mathbf{1})$ and $(\mathbf{4}, \mathbf{2})$. In the IR the metric is taken to have $S O(1,2) \times S O(2)_{D}$ symmetry. The twisting involve "mixing" the $S O(2)$ group of the metric with an $S O(2)$ included in $S O(3)_{R}$. This mixing is responsible for the breaking of some supersymmetries because the only spinors that can be defined on the curved manifold are those that have scalar properties on the curved part. In fact, it breaks $1 / 2$ of the supersymmetries of the five-dimensional SCFT.

Below we construct a gravity dual to the five-dimensional SCFT theory by finding a solution of Romans' theory with excited $F_{\mu \nu}^{I}$ fields. Then, we find a fixed-point solution for the massive field configuration. We postpone the study of the massless case to the 
next section.

Let us consider the metric ansätze of the form

$$
\begin{gathered}
d s^{2}=\mathrm{e}^{2 f}\left(d t^{2}-d r^{2}-d z^{2}-d v^{2}\right)-\frac{\mathrm{e}^{2 h}}{y^{2}}\left(d x^{2}+d y^{2}\right), \\
d s^{2}=\mathrm{e}^{2 f}\left(d t^{2}-d r^{2}-d z^{2}-d v^{2}\right)-\mathrm{e}^{2 h}\left(d \theta^{2}+\sin ^{2} \theta d \varphi^{2}\right),
\end{gathered}
$$

for $A d S_{4} \times H_{2}$ and $A d S_{4} \times S^{2}$, respectively. The only non-vanishing non-Abelian gauge potential components are taken to be

$$
A_{x}^{(3)}=\frac{a}{y} \quad, \quad A_{\varphi}^{(3)}=-a \cos \theta
$$

in the hyperbolic and spherical cases, respectively. One can treat both cases together by introducing a parameter $\lambda$ that can take two values

$$
\lambda=+1 \rightarrow H_{2}, \quad \lambda=-1 \rightarrow S^{2}
$$

We impose the following projections

$$
\gamma_{45}\left(T^{(3)}\right)_{i}^{j} \epsilon_{j}=\frac{\lambda}{2} \epsilon_{i}, \quad \gamma_{2} \gamma_{7} \epsilon_{i}=\epsilon_{i}
$$

A solution must satisfy the equations obtained by setting to zero the supersymmetry transformations for gauginos and gravitinos. After some algebra, they lead to

$$
\begin{aligned}
\varphi^{\prime} & =\frac{\mathrm{e}^{f}}{4 \sqrt{2}}\left[g \mathrm{e}^{\varphi}-3 m \mathrm{e}^{-3 \varphi}+2 a \lambda \mathrm{e}^{-2 h-\varphi}\right], \\
h^{\prime} & =\frac{\mathrm{e}^{f}}{4 \sqrt{2}}\left[-g \mathrm{e}^{\varphi}-m \mathrm{e}^{-3 \varphi}+6 a \lambda \mathrm{e}^{-2 h-\varphi}\right],
\end{aligned}
$$

and

$$
f^{\prime}=-\frac{\mathrm{e}^{f}}{4 \sqrt{2}}\left[g \mathrm{e}^{\varphi}+m \mathrm{e}^{-3 \varphi}+2 a \lambda \mathrm{e}^{-2 h-\varphi}\right],
$$

where $\varphi \equiv \phi / \sqrt{2}$.

A fixed-point solution of these equations is given by

$$
\begin{aligned}
\mathrm{e}^{4 \varphi} & =\frac{2 m}{g}, \\
\mathrm{e}^{-2 h} & =\sqrt{\frac{g m}{8}} \frac{1}{a \lambda}, \\
\mathrm{e}^{-f(r)} & =\frac{g \mathrm{e}^{\varphi}}{2 \sqrt{2}} r .
\end{aligned}
$$


In fact, it only happens if $\lambda a>0$. This solution satisfies the second order equations derived from the starting six-dimensional Lagrangian.

We can analyze the structure of the solutions of the system above near the UV of the theory $(r=0)$. Indeed, we can see that an expansion leads to the following behavior for the different fields

$$
f \approx-\log (r)+c_{1} r^{2}+\ldots, \quad g \approx-\log (r)+c_{2} r^{2}+\ldots, \quad \phi \approx c_{3} r^{2}+\ldots
$$

(where $c_{i}$ 's are constants). The interpretation of these expansions [27] is that the scalar field $\phi$ describes the coupling of the five-dimensional field theory to an operator of conformal weight $\Delta=3$ and mass squared $m^{2}=-6$. The operator is not the highest component of its supermultiplet, thus it is a deformation that breaks some supersymmetry.

The ten-dimensional metric corresponding to the fixed-point solution described above is easily obtained by using the results of [26],

$$
\begin{gathered}
X=\left(\frac{2 m}{g}\right)^{1 / 4}, \Delta=g X \cos ^{2} \chi+3 m X^{-3} \sin ^{2} \chi \\
d s_{10}^{2}=\left(3 m g^{2}\right)^{-1 / 8}(\sin \chi)^{\frac{1}{12}} X^{1 / 8}\left[\frac{\Delta^{3 / 8} \mathrm{e}^{2 f_{0}}}{2 r^{2}}\left(-d t^{2}+d r^{2}+d v^{2}+d z^{2}\right)\right. \\
+\frac{\sqrt{2} \Delta^{3 / 8}}{\sqrt{g^{3} m} y^{2}}\left(d x^{2}+d y^{2}\right)+\Delta^{3 / 8} \sqrt{2 m g^{3}} d \chi^{2} \\
\left.+\frac{\Delta^{-5 / 8}}{g X} \cos ^{2} \chi\left(\left(\sigma_{1}-\frac{d x}{y}\right)^{2}+\sigma_{2}^{2}+\sigma_{3}^{2}\right)\right]
\end{gathered}
$$

The metric has symmetries of the form $S O(2) \times S O(3)$. The corresponding expression for the RR fields and the ten-dimensional dilaton can be read from [26] and we do not quote it here.

Although we could not find an exact solution, we can carry out a similar analysis to [10]. Actually, the two cases $\lambda= \pm 1$ can be gathered again. Thus defining $F \equiv$ $u^{2} e^{-2 \varphi}, u \equiv e^{2 h(r)}$ and using Eqs.(22), (23) we obtain the following differential equation

$$
\frac{d F}{d u}=\left(\frac{3 g u^{4}-m F^{2}-10 a \lambda u F}{g u^{4}+m F^{2}-6 a \lambda u F}\right) \frac{F}{u} .
$$

For the case $\lambda=+1$ (hyperbolic plane), we can solve this equation in the approximations when $u \rightarrow \infty$ and $u \rightarrow 0$. In fact, in the UV one has

$$
F \approx u^{2}+\ldots, \quad \mathrm{e}^{-2 \varphi} \approx 1+\ldots
$$


and the metric results to be the $A d S_{6}$ limit of our original metric. In the IR we have

$$
F \approx F_{0} u^{5 / 3}+\ldots, \quad e^{-2 \varphi} \approx F_{0} u^{-1 / 3}+\ldots
$$

and the metric is

$$
d s_{6}^{2}=\frac{1}{u^{1 / 3}}\left(d t^{2}-d z^{2}-d v^{2}\right)-\frac{u}{y^{2}}\left(d x^{2}+d y^{2}\right)-\frac{2}{9 F_{0} a u^{1 / 3}} d u^{2} .
$$

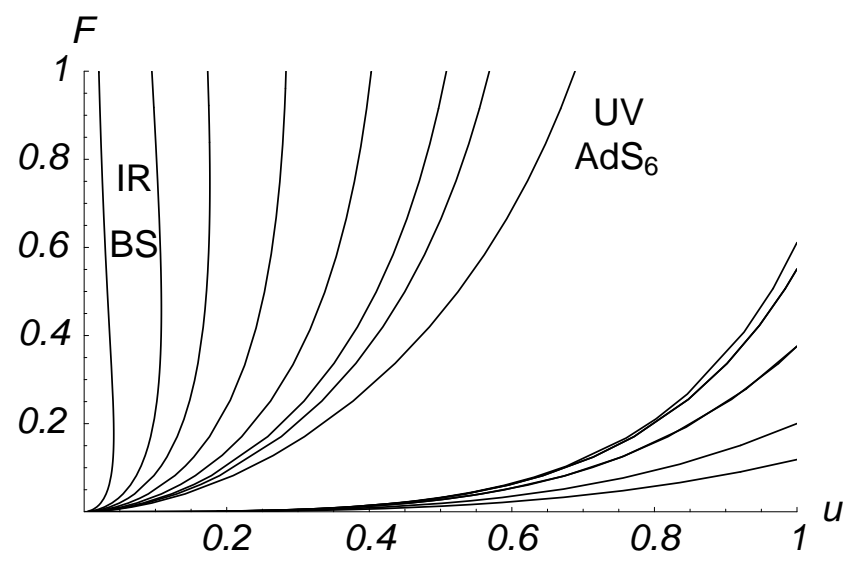

Figure 1: Orbits for the case $A d S_{4} \times H_{2}$. In the UV limit $F$ behaves as $u^{2}$ leading to an $A d S_{6}$-type region. On the other hand, it flows to "bad" singularities (BS) in the IR. We have used $g=3 m$ with $m=\sqrt{2}$, and $a=1$.

When up-lifted the singularities turn out to be of the "bad"type. They correspond to the curves flowing to the origin of the plots in figure 1. On the other hand, in both cases of the hyperbolic plane and sphere there are other kinds of "bad" singularities that correspond to the orbits going like $F \approx \frac{1}{u}$ as $u$ approaches to zero. Particularly, one can see this kind of behavior in figure 2. For the two-sphere we have the set of orbits depicted in figure 2 .

This also constitutes a part of the IR behavior of the flow. As we can see in figures 1 and 2, there is a smooth solution interpolating between the UV and the IR limits of the system, thus realizing our initial set up. The fixed-point solution is out of the range of this plot. 


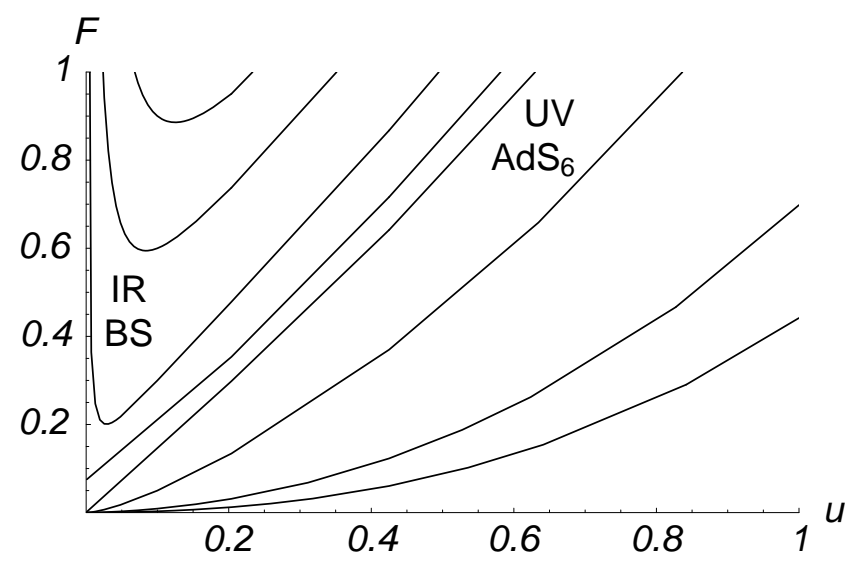

Figure 2: We show the behavior of the orbits for the case $S^{2}$. Similar comments as in the previous case are also valid here. For small $u$-values we can see the region corresponding to $F \approx \frac{1}{u}$.

In summary, we have found a fixed-point solution for the hyperbolic plane. In the UV both geometries flow to their corresponding asymptotic $A d S_{6}$. In the IR limit all the singularities in both geometries are of "bad" type according the criterion of ref. [8]. Similar behaviour is expected for the sphere. Note that the solutions studied here only represent a special case of solutions that, in general, have the form of a Laurent series. The fact that these solutions have non-acceptable singularities can be easily seen from the construction of an effective potential corresponding to this effective four dimensional system, and following the analysis outlined by Gubser in ref. [28] one can see that these kinds of solutions will not be interpretable as the IR of a gauge theory. However, this does not exclude the possibility of finding a different solution that could have an interpretation as the transcendental solution found in ref. [10].

\subsection{A non-Abelian solution in massive type IIA theory}

In this section we describe a non-Abelian solution in the massive IIA theory. Therefore, we consider a gravity dual of a five-dimensional SCFT with 8 supercharges that flows through the dimensions to a two-dimensional $(1,1)$ CFT with two supercharges at the IR. Its field content is given by a real scalar, a gauge field and the corresponding fermionic partner. The idea is to find a compactification from six-dimensional supergravity on $A d S_{3} \times H_{3}$. Under $S O(1,4) \times S O(3)_{R}$ the charges transform as $(\mathbf{4}, \mathbf{2})$ and $(\overline{\mathbf{4}}, \mathbf{2})$. After the twisting $S O(1,4) \times S O(3)_{R} \rightarrow S O(1,1) \times S O(3) \times S O(3)_{R} \rightarrow$ $S O(1,1) \times S O(3)_{D}$ we are left with two supercharges which are scalars under the diagonal subgroup. $S O(3)_{D}$ results from the identification of the normal bundle of the D4-brane world-volume with the spin bundle of $\Sigma_{3}$. They are the two "twisted" su- 
percharges that survive the twisting process. The field content of the theory can be obtained along the same lines and the result is the one mentioned above.

We believe that this solution is the first example of a non-Abelian solution of massive type IIA theory. It is similar to the solution obtained in [10] for massive sevendimensional gauged supergravity. In that case the solution up-lifts to $\mathrm{M}$ theory. Therefore, this suggests some connection between theories in the line of ref. [31] that merits further exploration.

Our configuration is

$$
d s^{2}=\mathrm{e}^{2 f}\left(d t^{2}-d r^{2}-d u^{2}\right)-\frac{\mathrm{e}^{2 h}}{y^{2}}\left(d x^{2}+d z^{2}+d y^{2}\right), \quad A_{x}^{(1)}=\frac{a}{y}, A_{z}^{(3)}=\frac{b}{y},
$$

we can also consider an $A d S_{3} \times S^{3}$, for $\lambda=-1$. The projections (all the indexes are plane indexes) are given by

$$
\gamma_{65}\left(T^{(3)}\right)_{i}^{j} \epsilon_{j}=\frac{1}{2} \epsilon_{i}, \quad \gamma_{64}\left(T^{(2)}\right)_{i}^{j} \epsilon_{j}=\frac{1}{2} \epsilon_{i}, \quad \gamma_{45}\left(T^{(1)}\right)_{i}^{j} \epsilon_{j}=\frac{1}{2} \epsilon_{i}, \quad \gamma_{7} \gamma_{2} \epsilon_{i}=\epsilon_{i}
$$

we use $\xi=e^{\frac{\phi}{\sqrt{2}}}$. The BPS equations are

$$
\begin{gathered}
a b=\frac{1}{g}, \\
h^{\prime}=-\frac{\mathrm{e}^{f}}{4 \sqrt{2}}\left[g \xi+m \xi^{-3}-10 \lambda a \xi^{-1} \mathrm{e}^{-2 h}\right], \\
\phi^{\prime}=\frac{\mathrm{e}^{f}}{4 \sqrt{2}}\left[g \xi-3 m \xi^{-3}+6 \lambda a \xi^{-1} \mathrm{e}^{-2 h}\right], \\
f^{\prime}=-\frac{\mathrm{e}^{f}}{4 \sqrt{2}}\left[g \xi+m \xi^{-3}+6 \lambda a \xi^{-1} \mathrm{e}^{-2 h}\right] .
\end{gathered}
$$

A fixed-point solution is obtained and it reads as it follows

$$
\mathrm{e}^{-2 h}=\frac{\sqrt{g^{3} m}}{2 \sqrt{6}}, \quad \xi=\left(\frac{3 m}{2 g}\right)^{1 / 4}
$$

This configuration can be uplifted to ten dimensions (massive IIA theory) using the results in $[26]$

$$
\begin{gathered}
X=e^{\phi / \sqrt{2}}, \Delta=g X \cos ^{2} \chi+3 m X^{-3} \sin ^{2} \chi \\
d s_{10}^{2}=\left(\frac{1}{3 m g^{2}}\right)^{1 / 8}(\sin \chi)^{\frac{1}{12}} X^{1 / 8}\left[\frac{\Delta^{3 / 8}}{2 r^{2}}\left(-d t^{2}+d r^{2}+d v^{2}\right)\right. \\
+\frac{\mathrm{e}^{2 h}}{2 y^{2}} \Delta^{3 / 8}\left(d x^{2}+d z^{2}+d y^{2}\right)+g^{2} \xi \Delta^{3 / 8} d \chi^{2} \\
\left.+\frac{\Delta^{-5 / 8}}{g X} \cos ^{2} \chi\left(\left(\sigma_{1}-\frac{d x}{y}\right)^{2}+\sigma_{2}^{2}+\left(\sigma_{3}-\frac{d z}{y}\right)^{2}\right)\right]
\end{gathered}
$$


where $\sigma_{i}$ are the three left-invariant forms in the three-sphere. We can see that the metric has an $S O(2) \times S O(3)$ invariance.

Since we are not able to integrate the above BPS system, we will repeat the analysis of the singularities as we did in the previous section. Again, both cases (hyperbolic plane and sphere) are treated together by using the parameter $\lambda= \pm 1$. Proceeding as above we get the following first order differential equation

$$
\frac{d F}{d u}=\left(\frac{3 g u^{4}-m F^{2}-14 a \lambda u F}{g u^{4}+m F^{2}-10 a \lambda u F}\right) \frac{F}{u} .
$$

When $\lambda=+1$ we solve this equation in two approximations, $u \rightarrow \infty$ and $u \rightarrow 0$. The large- $u$ approximation leads to the corresponding asymptotic $A d S_{6}$ whereas the other case gives

$$
\begin{gathered}
F \approx F_{0} \frac{1}{u}+\ldots, \quad \mathrm{e}^{-2 \varphi} \approx \frac{F_{0}}{u^{3}}+\ldots, \\
F \approx-2 \frac{a}{m} u+\ldots, \quad e^{-2 \varphi} \approx-\frac{2 a}{m u}+\ldots \\
F \approx F_{0} u^{7 / 5}+\ldots, \quad e^{-2 \varphi} \approx F_{0} u^{3 / 5}+\ldots .
\end{gathered}
$$

Note that all the singularities are of the "bad" type in the IR.

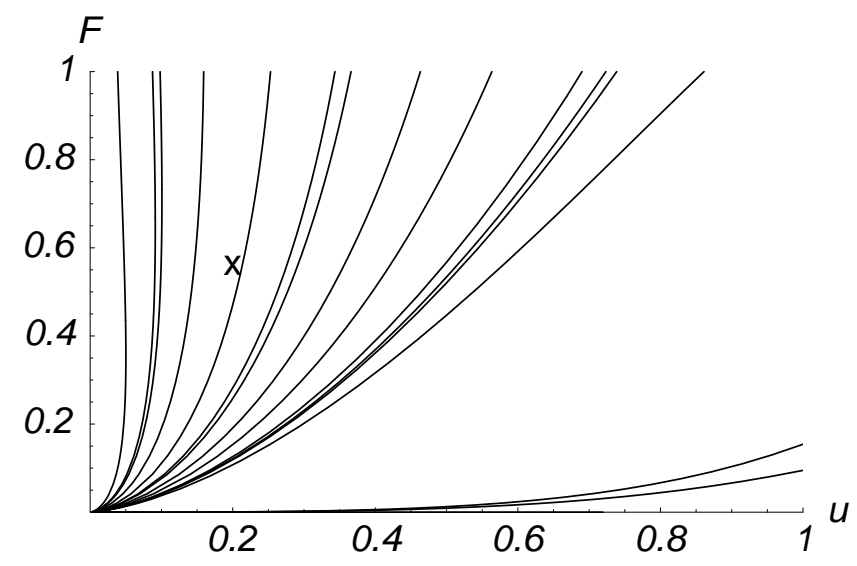

Figure 3: Behavior of the orbits for the non-Abelian massive case. The $A d S_{6}$-type region is at the UV, i.e., for large $u$ and $F$-values. In the IR it flows to "bad" singularities (BS). The cross indicates the fixed point-solution aforementioned. We again set $g=3 m, m=\sqrt{2}$, and $a=b=\lambda=1$.

Here we should make a similar comment as the one in the previous section: Gubser's criteria tells us to construct the effective potential of the three-dimensional gravity 
theory. With this potential we can see that the solutions in the form of a Laurent series showed above will not have an interpretation as the IR limit of a gauge theory.

\section{The massless case}

Consider six-dimensional gauged supergravity with the mass parameter taken to be zero $(m=0)$. We would like to find a ten-dimensional Kaluza-Klein ansatz which allows us to perform the up-lifting of certain solutions of the six-dimensional Romans' theory. In this section, we will show that the $\mathcal{N}=\tilde{4}^{g}$ theory can be embedded in type IIA theory.

Thus, we start with type IIA theory in ten dimensions and set the two- and threeform field strengths to zero. After the truncation the bosonic Lagrangian is

$$
\hat{\mathcal{L}}_{10}=\hat{R} * 1-\frac{1}{2} * d \hat{\phi} \wedge d \hat{\phi}-\frac{1}{2} \mathrm{e}^{-\frac{1}{2} \hat{\phi}} * \hat{F}_{(4)} \wedge \hat{F}_{(4)}
$$

The ten-dimensional equations of motion are

$$
\begin{aligned}
d \hat{*} d \hat{\phi} & =-\frac{1}{4} \mathrm{e}^{-\frac{1}{2} \hat{\phi}} \hat{*} \hat{F}_{(4)} \wedge \hat{F}_{(4)}, \\
d\left(\mathrm{e}^{\left.-\frac{1}{2} \hat{\phi} \hat{*} \hat{F}_{(4)}\right)}\right. & =0 \\
\hat{R}_{\mu \nu} & =\frac{1}{2} \partial_{\mu} \hat{\phi} \partial_{\nu} \hat{\phi}+\frac{1}{12} \mathrm{e}^{-\frac{1}{2} \hat{\phi}}\left(\hat{F}_{\mu \rho \sigma \tau} \hat{F}_{\nu}^{\rho \sigma \tau}-\frac{3}{32} g_{\mu \nu} \hat{F}_{(4)}^{2}\right), \\
\hat{F}_{(4)} \wedge \hat{F}_{(4)} & =0 .
\end{aligned}
$$

Notice that the last equation is a constraint resulting from the fact that we set $\hat{F}_{(3)}=$ $\hat{\mathcal{F}}_{(2)}=0$.

Reducing the theory on a circle, we get a nine-dimensional theory whose bosonic sector consists of a graviton, two scalars, a three-form field strength and a two-form field strength. One can consistently truncate this theory to a theory whose bosonic content includes a graviton, a scalar and a three-form field strength. The ansätze for the metric, scalar and three-form fields are

$$
\begin{aligned}
d \hat{s}_{10}^{2} & =\mathrm{e}^{-\frac{5}{8 \sqrt{14}} \bar{\phi}} d \bar{s}_{9}^{2}+\mathrm{e}^{\frac{35}{\sqrt{14}} \bar{\phi}} d Z^{2}, \\
\hat{\phi} & =\frac{\sqrt{14}}{8} \bar{\phi} \\
\hat{F}_{(4)} & =\bar{F}_{(3)} \wedge d Z .
\end{aligned}
$$

The resulting nine-dimensional Lagrangian and its equations of motion are

$$
\overline{\mathcal{L}}=\bar{R} * 1-\frac{1}{2} * d \bar{\phi} \wedge d \bar{\phi}-\frac{1}{2} \mathrm{e}^{-\frac{4}{\sqrt{14}} \bar{\phi}} * \bar{F}_{(3)} \wedge \bar{F}_{(3)},
$$




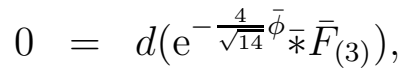

$$
\begin{aligned}
& \square \bar{\phi}=-\frac{1}{3 \sqrt{14}} \mathrm{e}^{-\frac{4}{\sqrt{14}} \bar{\phi}} \bar{F}_{(3)}^{2}, \\
& \bar{R}_{\mu \nu}=\frac{1}{2} \partial_{\mu} \bar{\phi} \partial_{\nu} \bar{\phi}+\frac{1}{4} \mathrm{e}^{-\frac{4}{\sqrt{14}} \bar{\phi}}\left(\bar{F}_{\mu \rho \sigma} \bar{F}_{\nu}^{\rho \sigma}-\frac{2}{21} g_{\mu \nu} \bar{F}_{(3)}^{2}\right) .
\end{aligned}
$$

Now, we compactify the 9-dimensional theory on $S^{3}$ using the formulae in [29]. The metric ansatz is

$$
d \bar{s}_{9}^{2}=X^{3 / 14} d s_{6}^{2}+\frac{1}{4 \tilde{g}^{2}} X^{-2 / 7} \sum_{i=1}^{3}\left(\sigma^{i}-\tilde{g} \tilde{A}_{(1)}^{i}\right)^{2},
$$

where $\sigma^{i}, i=1,2,3$ are left-invariant 1-forms of $S U(2)$

$$
\begin{aligned}
\sigma^{1} & =\cos \psi d \theta+\sin \psi \sin \theta d \varphi, \\
\sigma^{2} & =-\sin \psi d \theta+\cos \psi \sin \theta d \varphi, \\
\sigma^{3} & =d \psi+\cos \theta d \varphi
\end{aligned}
$$

and $X=\exp (\sqrt{2} \tilde{\phi})$. The ansätze for three-form field, its Hodge dual and the scalar field are

$$
\begin{aligned}
\mathrm{e}^{\sqrt{\frac{7}{2}} \bar{\phi}}= & X \\
\bar{F}_{(3)}= & \tilde{F}_{(3)}-\frac{1}{4 \tilde{g}^{2}} \tilde{h}^{1} \wedge \tilde{h}^{2} \wedge \tilde{h}^{3}+\frac{1}{4} \sum_{i=1}^{3} \tilde{F}_{(2)}^{i} \wedge \tilde{h}^{i}, \\
\mathrm{e}^{-\frac{4}{\sqrt{14}} \bar{\phi}} \bar{*} \bar{F}_{(3)}= & -\frac{1}{8 \tilde{g}^{3}} X^{-1} * \tilde{F}_{(3)} \wedge \tilde{h}^{1} \wedge \tilde{h}^{2} \wedge \tilde{h}^{3}+2 \tilde{g} X^{1 / 2} \epsilon_{(6)} \\
& +\frac{1}{16 \tilde{g}^{2}} \epsilon_{i j k} X^{-1 / 2} * \tilde{F}_{(2)}^{i} \wedge \tilde{h}^{j} \wedge \tilde{h}^{k},
\end{aligned}
$$

where $\tilde{h}^{i} \equiv \sigma^{i}-\tilde{g} \tilde{A}^{i}$. Substituting these ansätze into the nine-dimensional equations of motion we get

$$
\begin{aligned}
d \tilde{F}_{(3)} & =\frac{1}{2} \sum_{i=1}^{3} \tilde{F}_{(2)}^{i} \wedge \tilde{F}_{(2)}^{i}, \\
d\left(X^{-1} * \tilde{F}_{(3)}\right) & =0 \\
\mathcal{D}\left(X^{-1 / 2} * \tilde{F}_{(2)}^{i}\right) & =X^{-1} * \tilde{F}_{(3)} \wedge \tilde{F}_{(2)}^{i}, \\
d\left(X^{-1} * d X\right) & =X^{-1} * \tilde{F}_{(3)} \wedge \tilde{F}_{(3)}+\frac{1}{4} X^{-1 / 2} \sum_{i=1}^{3} * \tilde{F}_{(2)}^{i} \wedge \tilde{F}_{(2)}^{i}-4 \tilde{g}^{2} X^{1 / 2} \epsilon_{(6)} .
\end{aligned}
$$

The ansatz and the Lagrangian that produce Eq. (54) are

$$
d \hat{s}_{10}^{2}=\mathrm{e}^{\frac{\sqrt{2}}{8} \tilde{\phi}} d s_{6}^{2}+\frac{1}{4 \tilde{g}^{2}} \mathrm{e}^{-\frac{3 \sqrt{2}}{8} \tilde{\phi}} \sum_{i=1}^{3}\left(\sigma^{i}-\tilde{g} \tilde{A}_{(1)}^{i}\right)^{2}+\mathrm{e}^{\frac{5 \sqrt{2}}{8} \tilde{\phi}} d Z^{2},
$$




$$
\begin{aligned}
\hat{F}_{(4)} & =\left(\tilde{F}_{(3)}-\frac{1}{4 \tilde{g}^{2}} \tilde{h}^{1} \wedge \tilde{h}^{2} \wedge \tilde{h}^{3}+\frac{1}{4 \tilde{g}} \tilde{F}_{(2)}^{i} \wedge \tilde{h}^{i}\right) \wedge d Z, \\
\hat{\phi} & =\frac{1}{2 \sqrt{2}} \tilde{\phi}, \\
\frac{1}{\sqrt{g}} \mathcal{L}_{6} & =\tilde{R}-\frac{1}{2}(\partial \tilde{\phi})^{2}-\frac{1}{4} \mathrm{e}^{-\frac{1}{\sqrt{2}} \tilde{\phi}} \tilde{F}_{\mu \nu}^{i} \tilde{F}^{i \mu \nu}+4 \tilde{g}^{2} \mathrm{e}^{\frac{1}{\sqrt{2}} \tilde{\phi}}-\frac{1}{12} \mathrm{e}^{-\sqrt{2} \tilde{\phi}} \tilde{F}_{(3)}^{2},
\end{aligned}
$$

where $\tilde{F}_{(3)}=d \tilde{A}_{(2)}+\frac{1}{4}\left(\tilde{F}_{(2)}^{i} \wedge \tilde{A}_{(1)}^{i}-\frac{1}{6} \tilde{g} \epsilon_{i j k} \tilde{A}_{(1)}^{i} \wedge \tilde{A}_{(1)}^{j} \wedge \tilde{A}_{(1)}^{k}\right)$. For an easy comparison with the Romans' theory we make the following rescalings

$$
\tilde{g}_{\mu \nu} \rightarrow-\frac{1}{2} g_{\mu \nu}, \tilde{g} \rightarrow \frac{1}{2} g, \tilde{A}^{i} \rightarrow \sqrt{2} A^{i}, \tilde{\phi} \rightarrow 2 \phi, \tilde{F}_{3} \rightarrow F_{3}
$$

The Lagrangian and the ansatz in Eq.(54) now become

$$
\begin{aligned}
d \hat{s}_{10}^{2} & =-\frac{1}{2} \mathrm{e}^{\frac{\sqrt{2}}{4} \phi} d s_{6}^{2}+\frac{1}{g^{2}} \mathrm{e}^{-\frac{3 \sqrt{2}}{4} \phi} \sum_{i=1}^{3}\left(\sigma^{i}-\frac{1}{\sqrt{2}} g A_{(1)}^{i}\right)^{2}+\mathrm{e}^{\frac{5 \sqrt{2}}{4} \phi} d Z^{2}, \\
\hat{F}_{(4)} & =\left(F_{(3)}-\frac{1}{g^{2}} h^{1} \wedge h^{2} \wedge h^{3}+\frac{1}{\sqrt{2} g} F_{(2)}^{i} \wedge h^{i}\right) \wedge d Z, \\
\hat{\phi} & =\frac{1}{\sqrt{2}} \phi, \\
e^{-1} \mathcal{L}_{6} & =-\frac{1}{4} R+\frac{1}{2}(\partial \phi)^{2}-\frac{1}{4} \mathrm{e}^{-\sqrt{2} \phi} F_{\mu \nu}^{i} F^{i \mu \nu}+\frac{1}{8} g^{2} \mathrm{e}^{\sqrt{2} \phi}+\frac{1}{12} \mathrm{e}^{-2 \sqrt{2} \phi} F_{3}^{2},
\end{aligned}
$$

where $h^{i}=\sigma^{i}-\frac{1}{\sqrt{2}} g A^{i}$. The Lagrangian in Eq. (56) is precisely the $\mathcal{N}=\tilde{4}^{g}$ Romans' theory.

We can study a similar problem to the one in the previous section. One can write down a similar ansatz for the metric, i.e. a wrapped product of $A d S_{4} \times \Sigma_{2}$, where $\Sigma_{2}$ is a an hyperbolic plane or a sphere, as before. The system of Eqs.(22)-(24) can be studied in the massless case, i.e. $m=0$, while $g$ and $a$ are non-vanishing quantities. We have the solution

$$
f=-\varphi, \quad \varphi=\frac{g}{4 \sqrt{2}} r+\frac{1}{8} \log (r), \quad h=-\frac{g}{4 \sqrt{2}} r+\frac{3}{8} \log (r) .
$$

Therefore, the six-dimensional metric is given by

$$
d s^{2}=\frac{\mathrm{e}^{-\frac{g r}{2 \sqrt{2}}}}{r^{1 / 4}}\left[d t^{2}-d r^{2}-d z^{2}-d v^{2}-r d \Omega_{\lambda}^{2}\right] .
$$

The metric has a singularity. One way to resolve it is to look for a solution that has more degrees of freedom excited such as a non-Abelian solution. Another way to have an acceptable gravity solution is to construct a black hole solution such that the singularity is hidden by the horizon [21]. In the next section we will discuss a resolution of the singularity in the $S^{2}$ case, i.e., when the parameter $\lambda=-1$ in Eqs.(22) to (24). 


\subsection{A non-Abelian solution}

We would like to present a non-Abelian solution that resolves the "bad" singularity of the solution Eq.(58). This solution will be useful for the study of the gravity dual for the three-dimensional $\mathcal{N}=2$ super Yang-Mills theory. Actually this solution, being related to the solution in reference [9] represents a smeared NS-fivebrane on $S^{2}$ after a T-duality. In the IR the theory living on the brane will be $\mathcal{N}=2$ super YangMills theory in three dimensions plus Kaluza-Klein modes that do not decouple. The solution in the string frame is

$$
\begin{aligned}
d s^{2} & =2\left[d t^{2}-d r^{2}-d \vec{x}_{2}^{2}-\mathrm{e}^{2 h}\left(d \theta^{2}+\sin \theta^{2} d \varphi^{2}\right)\right], \\
F & =-w^{\prime} \sigma^{2} d r \wedge d \theta+w^{\prime} \sin \theta \sigma^{1} d r \wedge d \varphi+\left(w^{2}-1\right) \sigma^{3} \sin \theta d \theta \wedge d \varphi, \\
\phi_{S} & =\frac{1}{2} \log \left(\frac{\sinh (r)}{R(r)}\right), \quad w(r)=\frac{r}{\sinh (r)}, w^{\prime}=\frac{d w}{d r}, \\
\mathrm{e}^{2 h} & =R(r)^{2}, R(r)=\sqrt{2 r \operatorname{coth}(r)-r^{2} / \sinh (r)^{2}-1} .
\end{aligned}
$$

This is the same solution reported by Chamseddine and Volkov in $[32,33]$.

The relations between the scalars and metric in Einstein frame and string frame are

$$
\varphi=-\frac{1}{2} \phi_{S} ; \quad g_{\mu \nu}(E)=\mathrm{e}^{\phi_{S}} g_{\mu \nu}(S) .
$$

In Einstein frame the solution is

$$
d s_{E}^{2}=2 \mathrm{e}^{\phi_{S}}\left(-d t^{2}+d r^{2}+d \vec{x}_{2}^{2}\right)+\mathrm{e}^{2 G(r)}\left(d \theta^{2}+\sin ^{2} \theta d \varphi^{2}\right),
$$

where the dilaton is given by

$$
\begin{aligned}
\phi_{S} & =\frac{1}{2} \log \left(\frac{\sinh (r)}{R(r)}\right) \\
R(r) & =\sqrt{2 r \operatorname{coth}(r)-w(r)^{2}-1}, \quad \mathrm{e}^{2 G}=R(r)^{2}, \quad w(r)=\frac{r}{\sinh (r)} .
\end{aligned}
$$

The field strength components are

$$
\begin{aligned}
& F_{(2)}^{1}=w^{\prime} \sin \theta d r \wedge d \varphi \\
& F_{(2)}^{2}=-w^{\prime} d r \wedge d \theta, \\
& F_{(2)}^{3}=\left(w^{2}-1\right) \sin \theta d \theta \wedge d \varphi .
\end{aligned}
$$

Collecting all we have the following ten-dimensional solution:

$$
d \hat{s}_{10}^{2}=\mathrm{e}^{-\frac{3 \sqrt{2}}{8} \phi}\left[-d t^{2}+d r^{2}+d \vec{x}_{2}^{2}+\mathrm{e}^{2 G}\left(d \theta^{2}+\sin ^{2} \theta d \varphi^{2}\right)+\frac{1}{g^{2}} \sum_{i=1}^{3}\left(\sigma^{i}-\frac{g}{\sqrt{2}} A_{(1)}^{i}\right)^{2}\right]
$$




$$
\begin{aligned}
& +\mathrm{e}^{\frac{5 \sqrt{2}}{8} \phi} d Z^{2} \\
\hat{F}_{(4)}= & \left(-\frac{1}{g^{2}} h^{1} \wedge h^{2} \wedge h^{3}+\frac{1}{\sqrt{2} g} F_{(2)}^{i} \wedge h^{i}\right) \wedge d Z \\
\hat{\phi}= & \frac{1}{\sqrt{2}} \phi=-\frac{1}{4} \log \left(\frac{\sinh (r)}{R(r)}\right) .
\end{aligned}
$$

\section{A black hole solution in the massless theory}

The solutions of the massless six-dimensional Romans' theory, the string frame metric and 2-form ansatz are

$$
\begin{aligned}
d s^{2} & =\frac{f(r)}{r} d t^{2}-\frac{1}{r f(r)} d r^{2}-R^{2}\left(d \theta_{1}^{2}+\sin ^{2} \theta_{1} d \varphi_{1}^{2}\right)-d x^{2}-d y^{2}, \\
F_{2} & =h(r) d r \wedge d t+\frac{R Q_{m}}{\sqrt{2}} \sin \theta_{1} d \theta_{1} \wedge d \varphi_{1}
\end{aligned}
$$

where $f$ and $h$ are functions of $r$ while $\gamma$ and $Q_{m}$ are constants.

The solution in the string frame is

$$
h(r)=\frac{Q_{e}}{r^{2}}, \quad f(r)=-M+\frac{2 Q_{e}^{2}}{r}+\left(\frac{g^{2}}{2}+\frac{Q_{m}^{2}}{R^{2}}\right) r, \quad \phi(r)=\frac{1}{2} \log (r) .
$$

Similar solutions were previously discussed in [34]. Our solution has a different geometry and can carry both electric and magnetic charges.

One can up-lift the above solution to type IIA theory, and the results are

$$
\begin{aligned}
d \hat{s}_{10}^{2}= & -\frac{1}{2} r^{-1 / 8} \times \\
& \left(\frac{f(r)}{\sqrt{r}} d t^{2}-\frac{1}{\sqrt{r} f(r)} d r^{2}-R^{2} \sqrt{r}\left(d \theta_{1}^{2}+\sin ^{2} \theta_{1} d \varphi_{1}^{2}\right)-\sqrt{r}\left(d x^{2}+d y^{2}\right)\right) \\
& +\frac{r^{3 / 8}}{g^{2}}\left[\left(\sigma^{1}\right)^{2}+\left(\sigma^{2}\right)^{2}+\left(\sigma^{3}+\frac{g Q_{e}}{\sqrt{2} r} d t+\frac{g R Q_{m}}{2} \cos \theta_{1} d \varphi_{1}\right)^{2}\right]+r^{-5 / 8} d Z^{2}, \\
\hat{\phi}= & -\frac{1}{4} \log (r), \\
\hat{F}_{4}= & -\frac{1}{g^{2}} \sigma^{2} \wedge \sigma^{2} \wedge h^{3} \wedge d Z+\frac{1}{\sqrt{2} g} F_{2} \wedge h^{3} \wedge d Z \\
h^{3}= & \sigma^{3}+\frac{g Q_{e}}{\sqrt{2} r} d t+\frac{g R Q_{m}}{2} \cos \theta_{1} d \varphi_{1} .
\end{aligned}
$$

For different values of the constants $Q_{e}, Q_{m}, R, g$ we will have either a horizon or a naked singularity. It should be instructive to compute the entropy and the Hawking 
temperature of this black holes and comparing with the M-theory black holes obtained in [35]. Since the system analyzed in that reference is the same in string variables as the one we analyze here, it is expected that those results will be repeated. Indeed, the ten-dimensional interpretation suggested in [35] agrees with the one we have described above.

A solution with excited $B$-fields

Here we consider the six-dimensional Romans' theory with the mass parameter and all the fields except the scalar and the three-form field set to zero. In addition, we take $G_{3}$ to be a constant. The geometry of the $A d S_{3} \times R^{3}$ spacetime is given by

$$
d s^{2}=\mathrm{e}^{2 f}\left(d t^{2}-d r^{2}-d z^{2}\right)-\mathrm{e}^{2 h}\left(d x^{2}+d y^{2}+d v^{2}\right) .
$$

Let us consider a spinor satisfying the following constraints

$$
\gamma_{2} \gamma_{7} \epsilon_{i}=\epsilon_{i} \quad \gamma_{456} \epsilon_{i}=\epsilon_{i}
$$

and

$$
G_{x y v}=G
$$

From the vanishing of the supersymmetric variation of gravitinos and gauginos we obtain two independent equations

$$
\begin{aligned}
h^{\prime} & =-\mathrm{e}^{f}\left(\frac{g}{4 \sqrt{2}} \mathrm{e}^{\varphi}+\frac{G}{2} \mathrm{e}^{-3 h+2 \varphi}\right), \\
f^{\prime} & =-\mathrm{e}^{f}\left(\frac{g}{4 \sqrt{2}} \mathrm{e}^{\varphi}-\frac{G}{2} \mathrm{e}^{-3 h+2 \varphi}\right), \\
\varphi^{\prime} & =-h^{\prime} .
\end{aligned}
$$

A fixed-point solution can be easily obtained

$$
\mathrm{e}^{\frac{\phi}{\sqrt{2}}}=\frac{g \mathrm{e}^{3 h}}{2 \sqrt{2} G}
$$

The values of $h(r)$ remain undetermined since the equations for $\phi^{\prime}$ and $h^{\prime}$ are proportional to each other. In this case, $f(r)$ is given by

$$
f(r)=-\log \left(\frac{g^{2} \mathrm{e}^{3 h} r}{8 G}\right)
$$

therefore the metric is given by

$$
d s^{2}=\frac{1}{\left(\frac{g^{2} \mathrm{e}^{3 h} r}{8 G}\right)^{2}}\left(d t^{2}-d r^{2}-d z^{2}\right)-\mathrm{e}^{2 h}\left(d x^{2}+d y^{2}+d v^{2}\right) .
$$


Equation (72) implies that $\varphi=-h$, where for simplicity we omit integration constants. The system of equations above can be solved leading to

$$
f(h)=-h+\frac{1}{2} \log \left(\frac{g}{4 \sqrt{2}} \mathrm{e}^{4 h}+\frac{G}{2}\right) .
$$

Next, if we make the change of the integration variable $r \rightarrow h$, such that

$$
\mathrm{e}^{f} d r=-\frac{\mathrm{e}^{5 h}}{\left(\frac{g}{4 \sqrt{2}} \mathrm{e}^{4 h}\right)+\frac{G}{2}} d h
$$

in terms of $h$ the metric reads

$d s^{2}=-\frac{\mathrm{e}^{10 h}}{\left(\frac{g}{4 \sqrt{2}} \mathrm{e}^{4 h}+\frac{G}{2}\right)^{2}} d h^{2}+\mathrm{e}^{-2 h}\left(\frac{g}{4 \sqrt{2}} \mathrm{e}^{4 h}+\frac{G}{2}\right)\left(d t^{2}-d z^{2}\right)-\mathrm{e}^{2 h}\left(d x^{2}+d y^{2}+d v^{2}\right)$.

Using Eq.(16) we can write down the field strength of the dual theory as follows

$$
F_{t r z}=-G \mathrm{e}^{2 \sqrt{2} \phi+3 f-3 h} .
$$

The ten-dimensional solution is

$$
\begin{aligned}
d s_{10}^{2} & =-\frac{1}{2} \mathrm{e}^{\sqrt{2} \phi / 4} d s_{6}^{2}+\frac{1}{g^{2}} \mathrm{e}^{-3 \sqrt{2} \phi / 4}\left(d \theta^{2}+\sin \theta^{2} d \varphi^{2}+(d \psi+\cos \theta d \varphi)^{2}\right)+\mathrm{e}^{5 \sqrt{2} \phi / 4} d z_{1}^{2} \\
F_{4} & =-G \mathrm{e}^{2 \sqrt{2} \phi+3 f-3 h} d t \wedge d r \wedge d z \wedge d z_{1}-\frac{1}{g^{2}} \sin \theta d \psi \wedge d \theta \wedge d \varphi \wedge d z_{1} \\
\hat{\phi} & =\frac{1}{\sqrt{2}} \phi .
\end{aligned}
$$

The metric has a form of a warped product of a six-dimensional space, a three sphere and a single coordinate. We also note that the $G_{3}$ field does not play a similar role to the previous cases. In fact, there is no twisting in this case, since the space is not curved. One can view this solution as a NS-fivebrane of type IIB after wrapping one of the directions on a circle. It would be interesting to find an example in which the twisting on the curved manifold is performed by a $B_{2}$ field.

\section{Discussion}

Here we would like to start with some comments about the solutions presented in sections 3 and 4 . The solutions in section 3 are very similar to those obtained in [10] for

supergravities in seven dimensions. This may not be a coincidence since in the string 
frame both supergravities (one in seven dimensions and the other in six dimensions) have very similar structure. Furthermore, their supersymmetric transformations also look similar. This indicates that lower-dimensional supergravities are in some sense related to each other. This relation may be better understood in the light of the uplifted solutions in ten or eleven dimensions since the up-lifted solutions could be related by the known dualities.

In section 4 we constructed a non-Abelian solution that, as we noted, is identical to the solution obtained in $[32,33]$ and interpreted in [9] as a wrapped NS-fivebrane. In that case it was a gravity dual of a theory that is very similar to $\mathcal{N}=1 \mathrm{SYM}$. In our case, we can interpret the massless solution as the same NS-fivebrane with one direction compactified. Therefore, the number of supercharges remains the same and the world-volume theory is three-dimensional $\mathcal{N}=2 \mathrm{SYM}$ theory, as pointed out before. The identity of the string frame action in six dimensions (with $m=0$ ) and the action in seven dimensions (with topological mass equal to zero) reveals that we are dealing with the same system. We can extend this and try to look at the same issue in five dimensions. In fact, a similar situation occurs there too. One of the solutions presented in [36] can be related to the NS-fivebrane solution. In that paper, another solution with electric fields is shown. It would be interesting to understand this electromagnetic solution in the context of string theory.

We have analyzed the singularity structure of the equations representing D4-branes (of a D4-D8 system) wrapping two- and three-cycles. Because it was not possible for us to find exact solutions we turned to approximations inspired by ref. [10]. Thus, we could find some solutions in Laurent series. We argued that they were non-acceptable as the IR limit of a gauge theory. Expanding the solutions around $r=0$ we have identified the twisted theories on the brane-worldvolume and also found the operator inserted in the UV of the gauge theory, deforming it and breaking some supersymmetries. It is desirable to determine the precise form of the three (two) dimensional effective action of the SCFT that results after wrapping the brane on the two- and three-cycles.

It would be very nice to find new solutions where more fields are excited. For example solutions with the Abelian gauge field turned on could be interesting since it seems that they will involve a non-trivial $B_{2}$ field. It should also be useful to find solutions where the twisting is realized by a $B_{p}$ potential form (with $p>1$ ). The relevance of this type of solutions for non-commutative theories is evident. In this paper we found a solution in section 4 that has only $B_{2}$ fields excited. However, it does not represent a twisted theory since the brane is not wrapped on a curved manifold. Moreover, its ten-dimensional interpretation shows that we are dealing with a smeared NS-fivebrane.

The work of this paper together with the cases of M5, D3 and M2 branes constitute the cases that complete the flows between $A d S$-like spacetimes in the UV and the IR limit of gauge theories in lower dimensions. Finally, it would be interesting to 
study systems with compactifications like the ones discussed here but in the context of $F(4) \times G$ supergravity constructed in [37]. For that, it will be necessary to find a prescription for up-lifting this supergravity to ten dimensions. The analysis of those theories should give place to theories with enhanced $E_{N f+1}$ symmetry, and it should be very interesting to understand the relation between moduli spaces of Type I' and Heterotic strings from a gravitational point of view. 


\section{Acknowledgements}

We would like to thank Freddy A. Cachazo, José Edelstein, Jerome P. Gauntlett, Kentaro Hori, Andreas Karch, Nakwoo Kim, Ian I. Kogan, Juan Maldacena, Christopher N. Pope, Arta Sadrzadeh, Le G.Q. Thong and Cumrun Vafa for helpful discussions. The work of C.N. is supported by Fundación Antorchas of Argentina. The work of I.Y.P. is supported by the US Department of Energy under grant DE-FG0395ER40917. The work of M.S. is supported in part by funds provided by the U.S. Department of Energy (D.O.E.) under cooperative research agreement \#DF-FC0294ER40818, CONICET of Argentina, Fundación Antorchas of Argentina and The British Council. M.S. also acknowledges the kind hospitality of the Theoretical Physics of University of Oxford where part of this work was carried out.

\section{References}

[1] J. Maldacena, "The large N limit of superconformal field theories and supergravity," Adv. Theor. Math. Phys. 2 (1998) 231 [hep-th/9711200].

[2] S. S. Gubser, I. R. Klebanov and A. M. Polyakov, "Gauge theory correlators from non-critical string theory," Phys. Lett. B 428 (1998) 105 [hep-th/9802109].

[3] E. Witten, "Anti-de Sitter space and holography," Adv. Theor. Math. Phys. 2 (1998) 253 [hep-th/9802150].

[4] N. Itzhaki, J. M. Maldacena, J. Sonnenschein and S. Yankielowicz, "Supergravity and the large N limit of theories with sixteen supercharges," Phys. Rev. D 58 (1998) 046004 [hep-th/9802042].

[5] An extensive list of references can be found in K. Pilch and N. P. Warner, "N $=1$ supersymmetric renormalization group flows from IIB supergravity," hepth/0006066.

[6] D. Z. Freedman, S. S. Gubser, K. Pilch and N. P. Warner, "Renormalization group flows from holography supersymmetry and a c-theorem," hep-th/9904017.

[7] M. Bershadsky, C. Vafa and V. Sadov, "D-Branes and Topological Field Theories," Nucl. Phys. B 463 (1996) 420 [hep-th/9511222].

[8] J. Maldacena and C. Nunez, "Supergravity description of field theories on curved manifolds and a no go theorem," hep-th/0007018. 
[9] J. M. Maldacena and C. Nunez, "Towards the large n limit of pure $\mathrm{N}=1$ super Yang Mills," Phys. Rev. Lett. 86 (2001) 588 [hep-th/0008001].

[10] B. S. Acharya, J. P. Gauntlett and N. Kim, "Fivebranes wrapped on associative three-cycles," hep-th/0011190.

[11] J. P. Gauntlett, N. Kim and D. Waldram, "M-fivebranes wrapped on supersymmetric cycles," hep-th/0012195.

[12] H. Nieder and Y. Oz, "Supergravity and D-branes wrapping special Lagrangian cycles," hep-th/0011288.

[13] J. D. Edelstein and C. Nunez, "D6 branes and M-theory geometrical transitions from gauged supergravity," hep-th/0103167.

[14] K. Intriligator, D. R. Morrison and N. Seiberg, "Five-dimensional supersymmetric gauge theories and degenerations of Calabi-Yau spaces," Nucl. Phys. B 497 (1997) 56 [hep-th/9702198].

[15] A. Brandhuber and Y. Oz, "The D4-D8 brane system and five dimensional fixed points," Phys. Lett. B 460 (1999) 307 [hep-th/9905148].

[16] S. Ferrara, A. Kehagias, H. Partouche and A. Zaffaroni, "AdS(6) interpretation of 5D superconformal field theories," Phys. Lett. B 431 (1998) 57 [hep-th/9804006].

[17] L. J. Romans, "The F(4) Gauged Supergravity In Six-Dimensions," Nucl. Phys. B 269 (1986) 691.

[18] B. Janssen, P. Meessen and T. Ortin, Phys. Lett. B 453, 229 (1999) [hepth/9901078]. "D0-D8-F1 in massive IIA SUGRA," Phys. Lett. B 458 (1999) 283 [hep-th/9901136].

[19] W. Nahm, "Supersymmetries and their representations," Nucl. Phys. B 135 (1978) 149.

[20] B. S. DeWitt and P. van Nieuwenhuizen, "Explicit Construction Of The Exceptional Superalgebras F(4) And G(3)," J. Math. Phys. 23 (1982) 1953.

[21] A. Buchel, C. P. Herzog, I. R. Klebanov, L. Pando Zayas and A. A. Tseytlin, "Nonextremal gravity duals for fractional D3-branes on the conifold," hep-th/0102105. S. S. Gubser, C. P. Herzog, I. R. Klebanov and A. A. Tseytlin, "Restoration of chiral symmetry: A supergravity perspective," hep-th/0102172. A. Buchel, A. Frey, "Comments on supergravity dual of pure N=1 Super Yang Mills theory with unbroken chiral symmetry", hep-th/0103022. 
[22] For different studies in cosmologies of Randall-Sundrum models see for example L. Randall and R. Sundrum, "A large mass hierarchy from a small extra dimension," Phys. Rev. Lett. 83 (1999) 3370 [hep-ph/9905221]. L. Randall and R. Sundrum, "An alternative to compactification," Phys. Rev. Lett. 83 (1999) 4690 [hep-th/9906064]. I. I. Kogan, S. Mouslopoulos, A. Papazoglou, G. G. Ross and J. Santiago, Nucl. Phys. B 584 (2000) 313 [hep-ph/9912552]. L. Anchordoqui, C. Nunez and K. Olsen, "Quantum cosmology and AdS/CFT," JHEP0010 (2000) 050 [hep-th/0007064]. S. Nojiri, S. D. Odintsov and S. Zerbini, "Quantum (in)stability of dilatonic AdS backgrounds and holographic renormalization group with gravity," Phys. Rev. D 62 (2000) 064006 [hep-th/0001192]. S. W. Hawking, T. Hertog and H. S. Reall, "Brane new world," Phys. Rev. D 62 (2000) 043501 [hep-th/0003052]. J. M. Cline, C. Grojean and G. Servant, Phys. Rev. Lett. 83, 4245 (1999) [hep-ph/9906523].

[23] F. Giani, M. Pernici and P. van Nieuwenhuizen, "Gauged N=4 D =6 Supergravity," Phys. Rev. D 30 (1984) 1680.

[24] O. Aharony, A. Hanany, K. Intriligator, N. Seiberg and M. J. Strassler, "Aspects of $\mathrm{N}=2$ supersymmetric gauge theories in three dimensions," Nucl. Phys. B 499 (1997) 67 [hep-th/9703110].

[25] J. de Boer, K. Hori, H. Ooguri and Y. Oz, "Mirror symmetry in three-dimensional gauge theories, quivers and D-branes," Nucl. Phys. B 493 (1997) 101 [hepth/9611063]. J. de Boer, K. Hori, H. Ooguri, Y. Oz and Z. Yin, "Mirror symmetry in three-dimensional gauge theories, SL(2,Z) and D-brane moduli spaces," Nucl. Phys. B 493 (1997) 148 [hep-th/9612131]. J. de Boer, K. Hori and Y. Oz, "Dynamics of $\mathrm{N}=2$ supersymmetric gauge theories in three dimensions," Nucl. Phys. B 500 (1997) 163 [hep-th/9703100]. J. de Boer, K. Hori, Y. Oz and Z. Yin, "Branes and mirror symmetry in $\mathrm{N}=2$ supersymmetric gauge theories in three dimensions," Nucl. Phys. B 502 (1997) 107 [hep-th/9702154].

[26] M. Cvetic, H. Lu and C. N. Pope, "Gauged six-dimensional supergravity from massive type IIA," Phys. Rev. Lett. 83 (1999) 5226 [hep-th/9906221].

[27] V. Balasubramanian, P. Kraus and A. E. Lawrence, Phys. Rev. D 59 (1999) 046003 [hep-th/9805171].

[28] S. S. Gubser, "Curvature singularities: The good, the bad, and the naked," hepth/0002160.

[29] M. Cvetic, H. Lu and C. N. Pope, "Consistent Kaluza-Klein sphere reductions," Phys. Rev. D 62 (2000) 064028 [hep-th/0003286]. 
[30] F. A. Cachazo and C. Vafa, "Type I' and real algebraic geometry," hepth/0001029.

[31] I. V. Lavrinenko, H. Lu, C. N. Pope and K. S. Stelle, "Superdualities, brane tensions and massive IIA/IIB duality," Nucl. Phys. B 555 (1999) 201 [hepth/9903057].

[32] A. H. Chamseddine and M. S. Volkov, "Non-Abelian BPS monopoles in N $=4$ gauged supergravity," Phys. Rev. Lett. 79 (1997) 3343 [hep-th/9707176].

[33] A. H. Chamseddine and M. S. Volkov, "Non-Abelian solitons in $\mathrm{N}=4$ gauged supergravity and leading order string theory," Phys. Rev. D 57 (1998) 6242 [hepth/9711181].

[34] M. Cvetic, J. T. Liu, H. Lu and C. N. Pope, "Domain-wall supergravities from sphere reduction," Nucl. Phys. B 560 (1999) 230 [hep-th/9905096].

[35] D. Klemm, "BPS black holes in gauged $\mathrm{N}=4, \mathrm{D}=4$ supergravity," Nucl. Phys. B 545 (1999) 461 [hep-th/9810090].

[36] A. H. Chamseddine and M. S. Volkov, hep-th/0101202.

[37] R. D'Auria, S. Ferrara and S. Vaula, "Matter coupled F(4) supergravity and the AdS(6)/CFT(5) correspondence," JHEP0010 (2000) 013 [hep-th/0006107]. 